\title{
Modeling of passive constrained layer damping as applied to a gun tube
}

\author{
Margaret Z. Kiehl and C.P.T. Wayne Jerzak \\ Rensselaer Polytechnic Insitute, Department of \\ Mathematical Sciences, Amos Eaton 326, 110 8th \\ Street, Troy, NY 12180-3590, USA \\ Tel.: +1 518276 2307; E-mail: kiehim@rpi.edu
}

\begin{abstract}
We study the damping effects of a cantilever beam system consisting of a gun tube wrapped with a constrained viscoelastic polymer on terrain induced vibrations. A time domain solution to the forced motion of this system is developed using the GHM (Golla-Hughes-McTavish) method to incorporate the viscoelastic properties of the polymer. An impulse load is applied at the free end and the tip deflection of the cantilevered beam system is determined. The resulting GHM equations are then solved in MATLAB by transformation to the state-space domain.
\end{abstract}

\section{Introduction}

The production of long slender gun systems to meet increased exit velocity requirements of rounds has subsequently increased the effect of terrain induced vibrations. The increased susceptibility of long gun tubes to environmentally induced vibrations effects precision and accuracy of firing as well as barrel/round interactions during firing. A lightweight, low cost method is desired to damp terrain-induced vibrations thereby increasing performance of the gun system. A relatively inexpensive and lightweight method of damping vibrations in some structures is to apply a surface treatment of a viscoelastic material and a constraining layer. The viscoelastic material is bonded to the base material, a gun tube in our case, and to the constraining layer. Transverse vibrations in the system result in shear deformation of the viscoelastic material, which in turn dissipates the energy.

The integration of viscoelastic damping materials into various structures has been widely studied and various methods of modeling viscoelastic damping can be seen in the literature. Euler-Bernoulli beam theory has been used to model viscoelastic materials using the
Rayleigh Ritz approximation to damping. Timoshenko beam theory incorporates both shear deformation and rotational deformation in the formulation. The modal strain energy method [1] makes use of the relationship between damping factors and modal loss factors in order to assign damping factors to real elastic modes as an approximation to light damping. The Ross-KerwinUngar (RKU) theory uses continuum mechanics to derive a sixth order PDE for a three layer beam which incorporates damping using a complex shear modulus. The Golla-Hughes-McTavish (GHM) method [2, 3] uses a finite element approach where viscoelastic damping in introduced as a series of mini-oscillator terms and auxiliary dissipation coordinates.

DiTaranto and Blasingame [4] and Mead and Markus [5] derived a sixth order PDE to model the transverse vibrations of a three layer beam system based on the RKU equations developed for flexural vibrations of layered plates. In this approach damping of the viscoelastic layer is incorporated through the use of a complex shear modulus. Solutions of this equation in the frequency domain have lead successful predictions of loss factors and modal frequencies of a damped sandwich beam. Many investigators have used this approach in the study of sandwiched beams. Notably Miles and Reinhall [6] extended the theory to include thickness deformations, which allows for compression of the viscoelastic layer to contribute to the damping of the system. While this approach is stable in the frequency domain, the complex coefficients cause instability in the time domain.

Golla et al. [3] developed a finite element method approach to model constrained layer damping which is referred to as the GHM method. GHM starts from the framework of finite element analysis where the mass and stiffness matrixes are determined. Viscoelastic damping is incorporated into the model by replacing the constant material modulus with a stress-strain history law. Dissipation coordinates, which track the dissipation of energy, are then coupled with the physical coordinates of the model through a system of equations. Ro et al. [7] used this modeling technique in a layered 


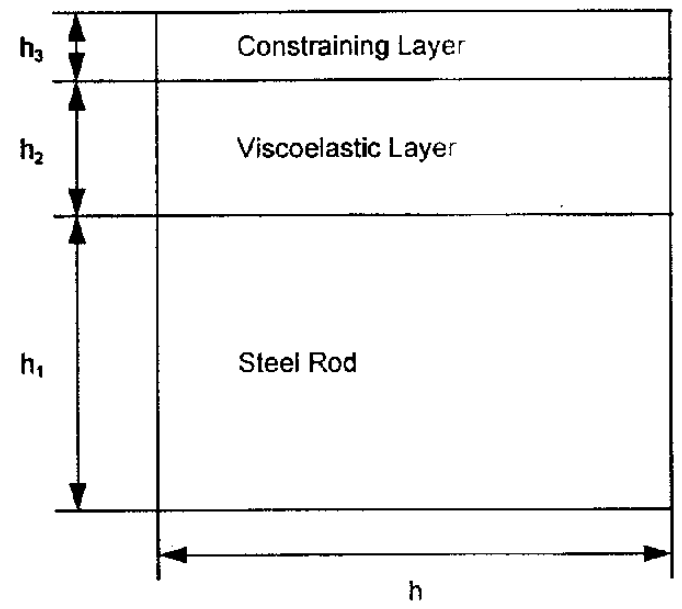

Fig. 1. Unbent three layer sandwich bar.

system to model active constrained layer damping of a gun tube with a moving load.

Given the need for a time domain solution, this study investigates the implementation of the GHM method in formulating a FEM model of a constrained layer gun tube.

\section{Viscoelastic constrained layer model}

A mathematical model of the system is determined by considering the equations of motion. We treat the gun tube as a long steel rod, coated with a layer of viscoelastic material and a thin constraining layer, Fig. 1. Assumptions are made that a perfect bond between the layers and that the sandwiched rod is much longer than it is thick. A cross section of the system consists of five layers, however, making full use of symmetry, the constrained rod can be modeled as a three-layer system.

As a transverse vibration passes along the rod, the layers of the system undergo varied deformation. Figure 2 depicts the deformation of the sandwich rod resulting from vibration. Due to the relative stiffness of the rod and constraining layer, the viscoelastic polymer undergoes shear deformation The material properties of the constraining layer under shear deformation provide for dissipation of energy and thus a damping of the vibration. In order to incorporate viscoeiastic properties into FEM, the GHM method uses a strain history constitutive relationship to develop the $\mathrm{M}$ and $\mathrm{K}$ matrices for the system. Viscoelastic material properties are incorporated directly into the mass and stiffness matrices, eliminating the need for complex coefficients

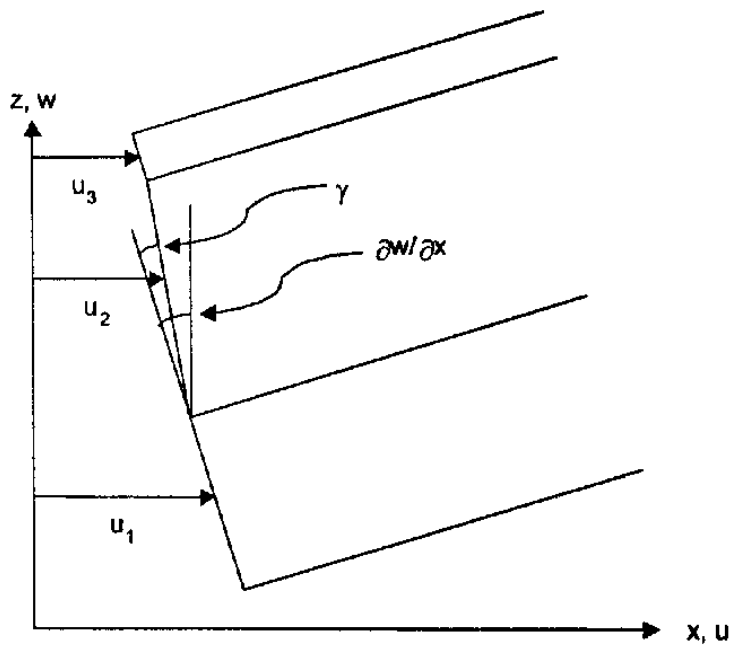

Fig. 2. Deflected bar.

which result in instability in the time domain. The viscoelastic properties are incorporated in the frequency domain through the use of 'minioscillators' [mh] to represent the material modulus of each layer. Transformation back into the time domain gives rise to the introduction of dissipation coordinates. The dissipation coordinates have no realization, but they are coupled to the real coordinates to account for the dissipation of energy from the system and are treated as additional degrees of freedom in the finite clement formulation. This approach is stable in the time domain and is shown to give plausible results for a single finite element of a three-layer rod.

\section{Finite element modeling}

The configuration of the sandwiched rod considered is that of a cantilevered beam, thus a finite element formulation for the time domain solution to the forced motion of a cantilever beam system is developed. The GHM method is employed to numerically compute the tip deflection of the cantilevered constrained layered rod for an impulse load applied at the free end. Assumptions made in using the GHM method are that 1) the core damping is linearly viscoelastic; 2) shear strains in the constraining layer and rod are negligible; 3 ) transverse displacements $w$ of each layer are equal; and 4) the constraining layer is to have no dissipative energy.

The sandwich rod is formulated into a finite element method (FEM) using one-dimensional beam elements 
with two nodal points, one at each end of the element. Four degrees of freedom are associated with each node. These are used to describe the longitudinal displacement $u_{3}$ of the constraining layer, the longitudinal displacement $u_{1}$ of the central rod, the transverse displacement $w$, and the slope ofthe displacement line $\partial w / \partial x$ (Recall Fig. 1). The basis vectors used to describe the longitudinal displacements are assumed to be linear polynomials, $b_{i}$ for $u_{3}$ and $\beta_{i}$ for $u_{1}$, and the basis vectors used for the transverse deflection are cubic Hermite polynomials, $\phi_{i}$. The resulting overall basis for a single beam element of length $h$ are given by:

$$
\begin{aligned}
b_{1} & =\beta_{1}=1-x / h, \\
b_{2} & =\beta_{2}=x / h, \\
\phi_{1} & =1-3(x / h)^{2}+2(x / h)^{3}, \\
\phi_{2} & =x-2 h(x / h)^{2}+h(x / h)^{3}, \\
\phi_{3} & =3(x / h)^{2}-2(x / h)^{3} \\
\phi_{4} & =-h(x / h)^{2}+h(x / h)^{3} .
\end{aligned}
$$

The generalized coordinate vector for a single beam element with initial starting value $x_{o}$ is

$$
\begin{aligned}
q= & {\left[u_{1}\left(x_{o}, t\right) u_{3}\left(x_{o}, t\right) w\left(x_{o}, t\right) w_{x}\left(x_{o}, t\right)\right.} \\
& u_{1}\left(x_{o}+h, t\right) u_{3}\left(x_{o}+h, t\right) w\left(x_{o}+h, t\right) \\
& \left.w_{x}\left(x_{o}+h, t\right)\right]^{T} .
\end{aligned}
$$

The use of the above basis and generalized coordinate vector for several beam elements comprising the system allows for the continuity of lateral displacements and transverse displacements and slopes across the elements.

The mass matrix $M_{i}$ and the stiffness matrix $K_{i}$ for the $i$ th element of the sandwich rod are obtained from the kinetic and potential energy, respectively. The kinetic and potential energies are determined by considering the contribution of each layer. By summing the contributions of each layer, the kinetic energy $T$ and corresponding mass matrix for the ith element become

$$
\begin{aligned}
T & =\frac{1}{2} \sum_{j=1}^{3} p_{j} A_{j} \int_{L_{i}}\left(\dot{w}^{2}+\dot{u}_{j}^{2}\right) d x \\
& =\frac{1}{2} q_{i}^{T} M_{i} q_{i}
\end{aligned}
$$

where the $j$ index indicates the $j$ th layer, $L_{i}$ is the length of the ith element, and the dot indicates a time derivative. In a similar fashion, the potential energy $V$ and associated stiffness matrix become

$$
\begin{aligned}
V= & \sum_{j=1}^{3} \frac{E_{j} A_{j}}{2} \int_{L_{i}}\left(\frac{\partial u_{j}}{\partial^{2} x}\right)^{2} d x \\
& +\sum_{j=1}^{3} \frac{E_{j} I_{j}}{2} \int_{L_{i}}\left(\frac{\partial^{2} w}{\partial^{2} x}\right)^{2} d x \\
& +\frac{G_{2} A_{2}}{2} \int_{L_{i}} \gamma^{2} d x \\
= & \frac{1}{2} q_{i}^{T} K_{i} q_{i}
\end{aligned}
$$

where $G_{2}$ is the viscoelastic shear modulus and $r$ is the shear strain. When the system is modeled using on beam element, the equation of motion for the system, analogous to the FEM formulation of an EulerBernoulli beam is given by

$$
M \ddot{q}+K q=f
$$

where $f$ is a vector containing the forcing terms.

\subsection{GHM method implementation}

Incorporation of the viscoelastic damping is implemented by first expressing the stiffness matrix as a sum of stiffness matrices each of which depend upon an individual layer:

$$
K=\sum_{j} K_{j}=\sum_{j} G_{j} \bar{K}_{j} .
$$

For each layer, the long-term behavior of the material modulus is then factored out of the corresponding stiffness matrix as $G_{j}$. A hereditary stress-strain law is then used to incorporate the viscoelastic damping. For illustration purposes, the development of the GHM viscoelastic element will first be presented for a oneelement/one layer beam. The general finite element equations of motion are of the same form as found in Eq. 5 with the appropriate mass and stiffness matrix for a viscoelastic layer and the material modulus factored out. The stressstrain law results in the following equations of motion

$$
M \ddot{q}(t)+\int_{o}^{t} G(t-\tau) K \dot{q}(\tau) d \tau=f(t) .
$$

This becomes

$$
s^{2} M \tilde{q}(s)+s \tilde{G}(s) \bar{K} \tilde{q}(s)=\tilde{f}(s)
$$

when transformed into the Laplace domain.

GHM uses a sum of a finite number of minioscillators terms to incorporate viscoelastic damping. Let us first consider the case of one mini-oscillator to 
define the material modulus. In the Laplace domain, this is represented as

$$
s \tilde{G}(s)=G^{\infty}\left[1+\alpha \frac{s^{2}+2 \xi \omega s}{s^{2}+2 \xi \omega s+\omega^{2}}\right]
$$

where $\alpha, \xi, \omega$ are positive constants. In particular. $\omega$ represents a model frequency while $\alpha$ and $\xi$ determine the shape of the material modulus function in the Laplace domain. A column vector of dissipation coordinates is introduced with the following behavior:

$$
\tilde{\hat{z}}(s)=\frac{\omega^{2}}{s^{2}+2 \xi \omega s+\omega^{2}} \tilde{q}(s) .
$$

Coupling the dissipation coordinates with Laplace domain equations of motions and converting to a second-order time domain realization, our resulting system of equations become

$$
\begin{aligned}
& {\left[\begin{array}{cc}
M & 0 \\
0 & \alpha \frac{1}{\omega^{2}} K
\end{array}\right]\left[\begin{array}{c}
\dot{q} \\
\dot{\hat{z}}
\end{array}\right]+\left[\begin{array}{cc}
0 & 0 \\
0 & \alpha \frac{2 \xi}{\omega}
\end{array}\right]\left[\begin{array}{c}
\dot{q} \\
\dot{\hat{z}}
\end{array}\right]} \\
& +\left[\begin{array}{cc}
K(1+\alpha)-\alpha K \\
-\alpha K & \alpha K
\end{array}\right]\left[\begin{array}{c}
q \\
\hat{z}
\end{array}\right] \\
& =\left[\begin{array}{c}
f \\
0
\end{array}\right] .
\end{aligned}
$$

In order to eliminate the rigid body modes from the stiffness matrix, spectral decomposition is used resulting in

$$
K=G^{\infty} \bar{K}=G^{\infty} \bar{R} \bar{\Lambda} \bar{R}^{T}
$$

where $\bar{\Lambda}$ is a diagonal matrix of nonzero eigenvalues of $\bar{K}$ and the columns of $\bar{R}$ are the corresponding orthonormalized eigenvectors. $G^{\infty}$ is then multiplied back into the diagonal matrix and the following substitutions, Eq (13), are made into Eq. (11).

$$
z=\bar{R}^{T} \hat{z} \quad R=\bar{\Lambda}
$$

Pre-multiplying the bottom row by $R^{T}$, the equations of motion are of the form:

$$
M_{v}\left[\begin{array}{c}
\ddot{q} \\
\ddot{z}
\end{array}\right]+D_{v}\left[\begin{array}{l}
\dot{q} \\
\dot{z}
\end{array}\right]+K_{v}\left[\begin{array}{l}
q \\
z
\end{array}\right]=\left[\begin{array}{l}
f \\
0
\end{array}\right]
$$

where the GHM matrices are

$$
\begin{aligned}
M_{v} & =\left[\begin{array}{cc}
M & 0 \\
0 & \alpha \frac{1}{\omega^{2}} \Lambda
\end{array}\right] \\
D_{v} & =\left[\begin{array}{cc}
0 & 0 \\
0 & \alpha \frac{2 \xi}{\omega} \Lambda
\end{array}\right] \\
K_{v} & =\left[\begin{array}{cc}
K(1+\alpha)-\alpha R \\
-\alpha R^{T} & \alpha \Lambda
\end{array}\right] .
\end{aligned}
$$

The GHM method can be extended to three layers with individual material properties. The mass and stiffness matrices for each individual layer are formulated as discussed above. Once the mass and stiffness matrices are then combined, the resulting equations of motion for the system once stress-strain history is applied become

$$
\begin{aligned}
& M \ddot{q}(t)+\int_{o}^{t}\left[\sum_{j} G_{j}(t-\tau) K_{j}\right] \dot{q}(\tau) d \tau \\
= & f(t) .
\end{aligned}
$$

Note that $j$ indexes the layer. Each of the material moduli, $G_{j}$, for which GHM is used to incorporate viscoelastic damping is written as a sum of a finite number of mini-oscillators terms, Using three mini-oscillator terms, the damping is represented in the Laplace domain as

$$
\begin{aligned}
& s \tilde{G}_{j}(s) \\
= & G_{j}^{\infty}\left[1+\sum_{k=1}^{3} \alpha_{j k} \frac{s^{2}+2 \xi_{j k} \omega_{j k} s}{s^{2}+2 \xi_{j k} \omega_{j k} s+\omega_{j k}^{2}}\right]
\end{aligned}
$$

where $\alpha_{j k}, \xi_{j k}, \omega_{j k}$ are positive constants associated with the $k$ th mini-oscillator for the $j$ th layer. For each minioscillator within a layer, a column vector of dissipation coordinates is introduced with the following behavior:

$$
\tilde{\hat{z}}(s)=\frac{\omega_{j k}^{2}}{s^{2}+2 \xi_{j k} \omega_{j k} s+\omega_{j k}^{2}} \tilde{q}(s) .
$$

Coupling the dissipation coordinates with Laplace domain equations of motions and converting to a second-order time domain realization in a way analogous to the presentation described above, our resulting system of equations become

$$
M_{v}\left[\begin{array}{c}
\ddot{q} \\
\ddot{z}
\end{array}\right]+D_{v}\left[\begin{array}{c}
\dot{q} \\
\dot{z}
\end{array}\right]+K_{v}\left[\begin{array}{l}
q \\
z
\end{array}\right]=\left[\begin{array}{l}
f \\
0
\end{array}\right] .
$$

Now the GHM viscoelastic matrix elements are of the form $2 \varepsilon_{j k} \omega_{j k}$, where the $j$ indexes the layer and the $k$ indexes the mini-oscillator.

\subsection{Space-state formulation}

Once these matrices have been formulated, the system can be transformed into a space-state formulation [8]. This is done by first defining the state-vector as follows:

$$
x=\left[\begin{array}{l}
r \\
\dot{r}
\end{array}\right] \text { where } r=\left[\begin{array}{l}
q \\
z
\end{array}\right] .
$$




$$
\begin{aligned}
M_{v} & =\left[\begin{array}{cccccc}
M & 0 & \ldots & \ldots & \ldots & 0 \\
0 & \alpha_{11} \frac{1}{\omega_{11}^{2}} \Lambda_{1} & 0 & \ldots & \ldots & 0 \\
\vdots & 0 & \ddots & 0 & \ldots & 0 \\
\vdots & \vdots & 0 & \alpha_{j k} \frac{1}{\omega_{j k}^{2}} \Lambda_{j} & 0 & 0 \\
\vdots & : & 0 & \ddots & 0 & \\
0 & 0 & 0 & 0 & 0 & \alpha_{33} \frac{1}{\omega_{33}^{2}} \Lambda_{3}
\end{array}\right] \\
D_{v} & =\left[\begin{array}{ccccccc}
0 & 0 & \ldots & \ldots & \ldots & 0 \\
0 & \alpha_{11} \frac{2 \xi_{11}}{\omega_{11}} \Lambda_{1} & 0 & \ldots & \ldots & 0 \\
\vdots & 0 & \ddots & 0 & \ldots & 0 \\
\vdots & \vdots & 0 & \alpha_{j k} \frac{2 \xi_{j k}}{\omega_{j k}} \Lambda_{j} & 0 & 0 \\
\vdots & \vdots & 0 & \ddots & 0 & \\
0 & 0 & 0 & 0 & 0 & \alpha_{33} \frac{2 \xi_{33}}{\omega_{33}} \Lambda_{3}
\end{array}\right] \\
K_{v} & =\left[\begin{array}{ccccccc}
K+\sum_{j, k} \alpha_{j k} K_{j} & -\alpha 11 R_{1} & \ldots & -\alpha_{j k} R_{j} & \ldots & -\alpha_{33} R_{3} \\
-\alpha 11 R_{1}^{T} & -\alpha 11 \Lambda_{1} & 0 & \ldots & \ldots & 0 \\
\vdots & & 0 & \ddots & 0 & \ldots & 0 \\
-\alpha_{j k} R_{j}^{T} & \vdots & 0 & \alpha j k \Lambda_{j} & 0 & 0 \\
-\alpha_{33} R_{3}^{T} & & 0 & 0 & 0 & 0 & \alpha 33 \Lambda_{3}
\end{array}\right]
\end{aligned}
$$

Equation 19 can then be posed in terms of $r$ and $\dot{r}$ as

$$
\begin{aligned}
& \dot{r}=I \dot{r} \\
& \ddot{r}=-M_{v}^{-1} K_{v} r-M_{v}^{-1} D_{v} \dot{r}+M_{v}^{-1} f .
\end{aligned}
$$

This can then be transformed into a format that is compatible with the capabilities of MATLAB ${ }^{\mathrm{TM}}$ by expressing the system as

$$
\begin{aligned}
& \dot{x}=A x+B u \\
& y=C x+D u
\end{aligned}
$$

where the matrices $A, B, C$, and $D$ are constructed as prescribed by the equations given below.

$$
\begin{aligned}
& A=\left[\begin{array}{cc}
0 & I \\
M_{v}^{-1} K_{v} & M_{v}^{-1} D_{v}
\end{array}\right] \\
& B=\left[\begin{array}{c}
0 \\
M_{v}^{-1}
\end{array}\right] \\
& C=\left[\begin{array}{ll}
I & 0
\end{array}\right]=[0] \\
& y=q \quad u=f
\end{aligned}
$$

This representation of the time domain problem can then be solved using the impulse command in MATLAB $^{\text {TM }}$.

\section{Model results}

The specifications for the sandwich rod modeled using a finite element formulation and GHM method are given here. The steel rod used to simulate the gun tube has an inner radius of $0.0222 \mathrm{~m}$ and an outer radius of $0.0445 \mathrm{~m}$. The density and Young's modulus are given by $7832 \mathrm{~kg} / \mathrm{m}^{3}$, and $207 \times 10^{9} \mathrm{~Pa}$ respectively. The constraining layer has the following properties: $p=1993 \mathrm{~kg} / \mathrm{m}^{3}, E=24.1 \times l 0^{9} \mathrm{~Pa}$, and thickness of $0.0005 \mathrm{~m}$. Sorbithane, the viscoelastic polymer, is characterized by a thickness of $0.003175 \mathrm{~m}$, density of $1281 \mathrm{~kg} / \mathrm{m}^{3}$, Poisson's ratio $v=0.49$, and bulk shear modulus and elastic modulus given by $G=0.3356 \times l 0^{6} \mathrm{~Pa}$ and $E=1 \times l 0^{6} \mathrm{pa}$. The moment of inertia, $I$, and cross sectional area. $A$, are calculated using the standard formulas $I=1 / 4 \pi r^{4} A=r^{2}$. The material parameters for the sandwich rod are taken from tables and manufacturer provided specifications.

In incorporating the GHM model, it is assumed that the shear strain the constraining layer is negligible. This results in modeling the steel rod and constraining layer as elastic medium while the polymer is considered to be linearly viscoelastic. Additionally, it is assumed that transverse displacements for any given cross section are uniformly constant for the entire system. As a 
A)

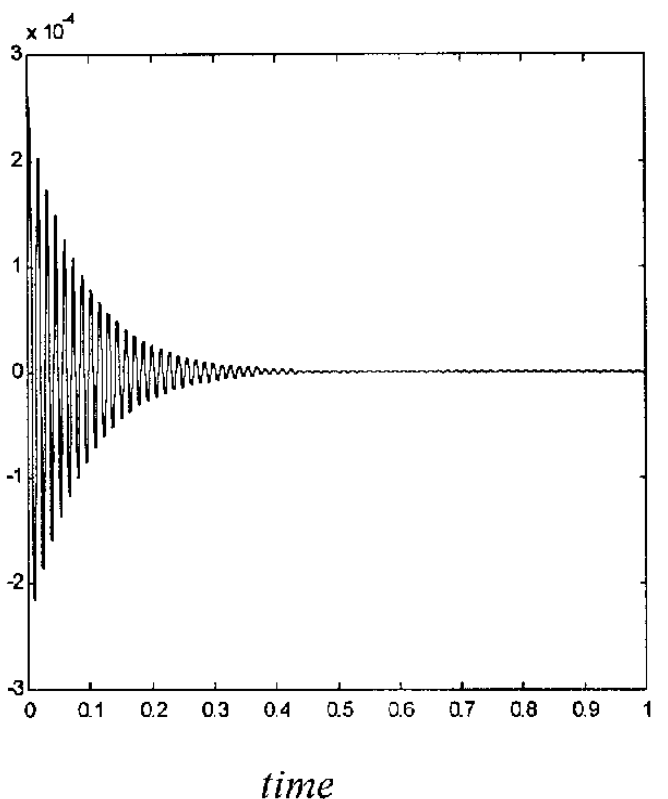

B)

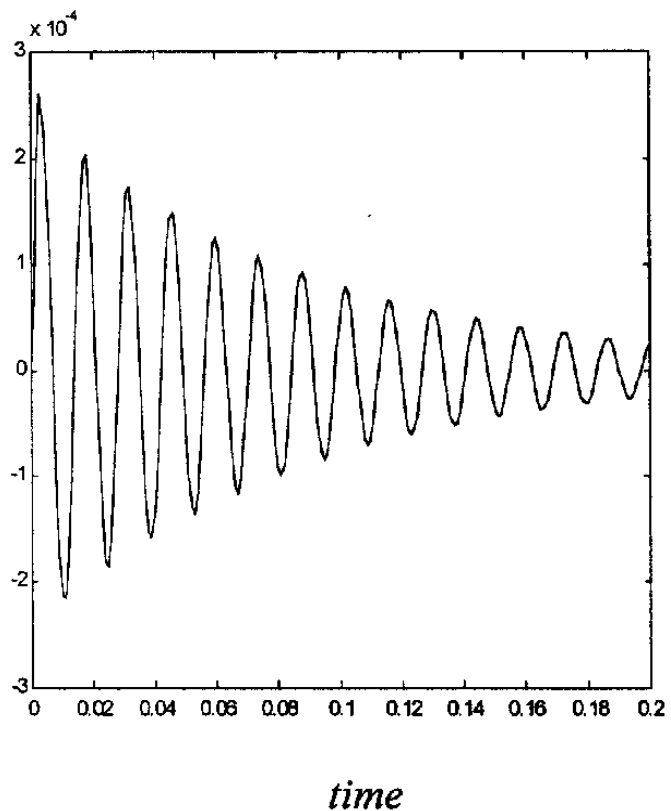

Fig. 3. Damping of cantilever beam subject to an impulse at the free end, where A) depicts long time behavior and B) depicts short time damped oscillations.

first approximation, the values for the material modulus shape functions $\alpha_{j k}, \xi_{j k}, \omega_{j k}$ reported by Ro et al. [7] are implemented. Curve fitting of experimental data will provide more accurate values for these parameters.

\section{Discussion and conclusion}

Our initial modeling of the time dc]main was done as a one element - three layer beam. This solution to the cantilever beam with impulse shock applied to the free end is given in Fig. 3. An implementation was also attempted for a three element - three layer beam model. The details of this model are still under investigation to obtain a converging finite element solution. The primary goal of any further research will be to successfully implement the three element - three layer beam model. This is key to the capability to model the gun tube using $n$ elements.

There are other things that need to be considered for future work in order to validate the model. There is a need to run frequency test on the viscoelastic polymer and gun tube in order to determine the appropriate values for $\alpha_{j}, \xi_{j}$, and $\omega$ for the $j$ modal frequencies we wish to use in this model. Data obtained from the frequency tests can then be curve fit using modal circles. Further steps towards verification of the model include construction of a constrained layer damped rod Impulses from a hammer could then be applied to this mechanical model and experimental results could be gathered. This would allow for the comparison of the FEM model to the experimental results.

The initial findings of the model are very encouraging. The implementation of GHM in order to incorporate viscoelastic damping allows for a stable time domain solution in the simplified case of a single finite element. Initial results indicate that a time domain solution for multiple finite elements will follow accordingly. Also, significant damping of the impulse applied to the tip of the sandwiched rod is seen using the model. This result is consistent with the application of a constrained viscoelastic layer.

\section{References}

[1] C.D. Johnson and D.A. Kienholz, Finite element prediction of Damping in Structures with Constrained Viscoelastic Layers, AAIA Journal 20(9) (1982), \#-\#.

[2] D.J. McTavish and P.C. Hughes, Modeling of Linear Viscoelastic Space Structures, Journal of Vibration and Acoustics $\mathbf{1 0 5}$ (1993), 103-110.

[3] D.H. Golla and P.C. Hughes, Dynamics of Viscoelastic Structures - A Time-Domain, Finite Element Formulation, Journal of Applied Mechanics 52 (1985), 897-906. 
[4] R.A. DiTaranto and W. Blasingame, Composite damping of vibrating sandwich beams, Journal of Engineering Ind. \# (1967), \#-\#.

[5] D.J. Mead and S. Markus, The Forced Vibration of a Threelayer, Damped Sandwich Beam with Arbitrary Boundary Conditions, Journal of Sound and Vibrations 10(2) (1969), 163175.

[6] R.N. Miles and P.G. Reinhall, An Analytical Model for the Vibration of Laminated Beams Including the Effects of both shear and thickness deformation in the adhesive layer, Journal of Vibration, Acoustics, Stress, and Reliability in Design $\mathbf{1 0 8}$ (1986), 56-64.
[7] J. Ro, K.S. El-Din and A. Baz, Vibration Control of Tubes With Internally Moving Loads Using Active Constrained Layer Damping, Asme Applied Mechanics Division -PublicationsAmd 223 (1997), 1-12.

[8] E.L. Kathe, MATLAB Modeling of Non-Uniform Beams Using the Finite Element Method for Dynamic Design and Analysis. US Amy Armaments Research, Development and Engineering Center; Close Combat Armaments Center, Benèt Laboratories; Technical Report ARCCB-TR-960 10, Watervliet, NY. 

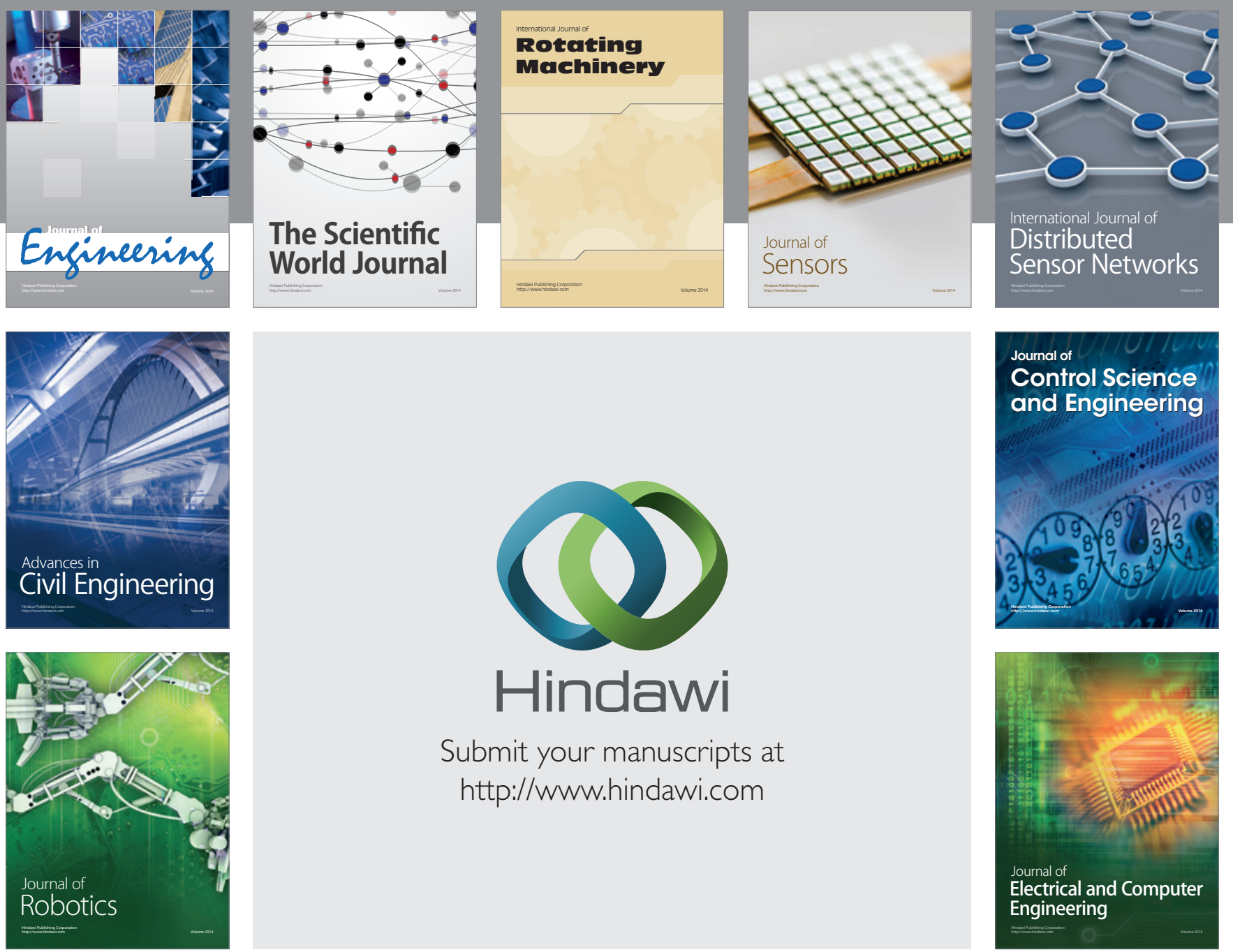

Submit your manuscripts at

http://www.hindawi.com
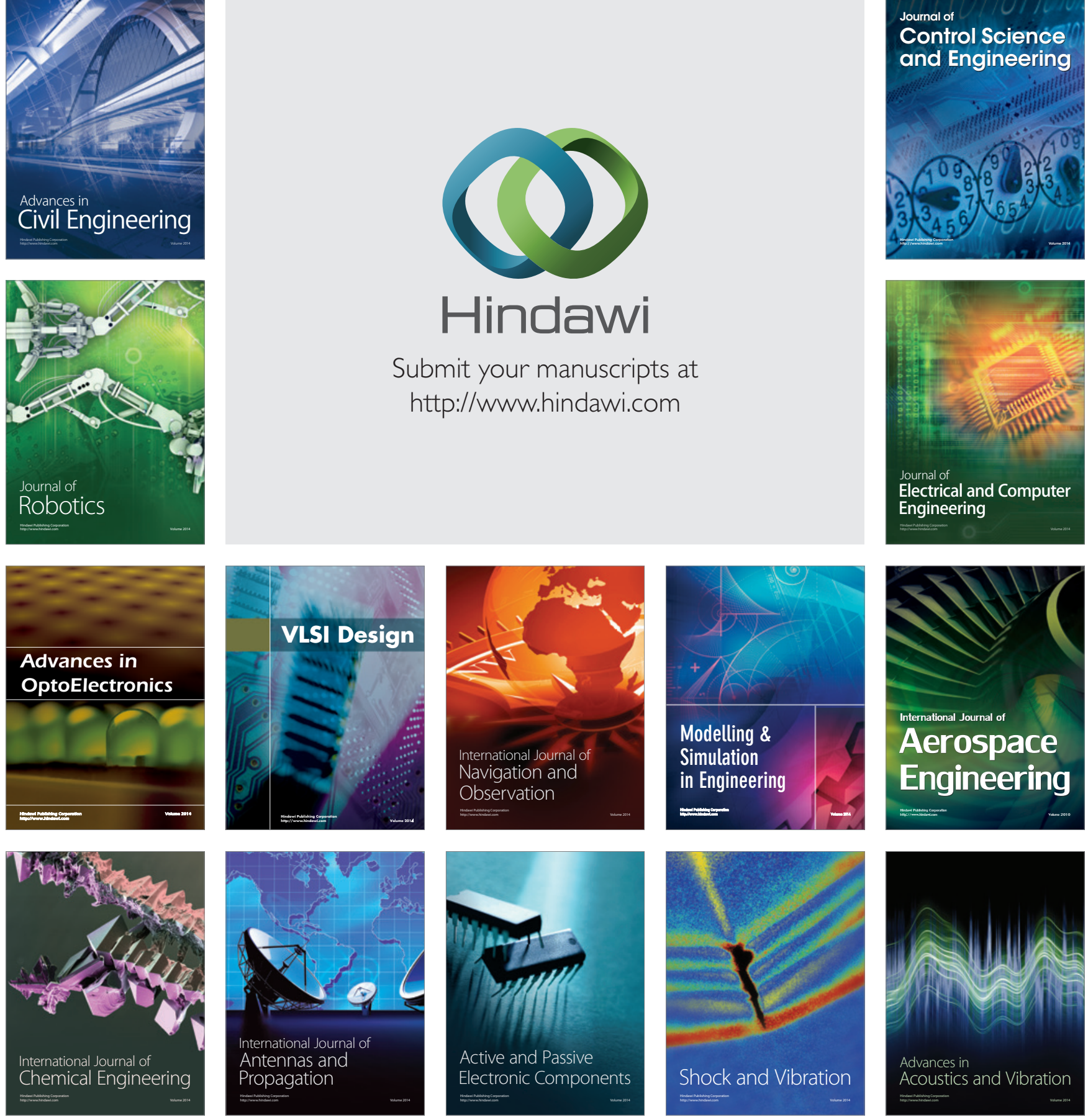